\title{
Application of Taguchi Robust Design for optimizing tensile strength response of Ukam-plant fibre reinforced cashew nut shell liquid (CNSL) composite by the effects of control parameters
}

\author{
Nwigbo Solomon Chuka ${ }^{1}$, Godwin Kingsley Obiazi 1, ${ }^{*}$ and Ikebudu Kingsley Okechukwu ${ }^{2}$ \\ ${ }^{1}$ Department of Mechanical Engineering, Nnamdi Azikiwe University, Awka. Nigeria. \\ ${ }^{2}$ Department of Mechanical Engineering, Odumegu Ojukwu University, Uli. Nigeria.
}

Global Journal of Engineering and Technology Advances, 2021, 08(01), 039-050

Publication history: Received on 11 April 2021; revised on 08 July 2021; accepted on 11 July 2021

Article DOI: https://doi.org/10.30574/gjeta.2021.8.1.0058

\begin{abstract}
This paper focuses on the application of Taguchi Robust Design to determine the optimum tensile strength of UkamPlant Fibre Reinforced CNSL Composite using indigenous or locally sourced vegetable or plant based natural fibres (Ukam-plant fibres) that are Lignocellulose as potential raw material. Tensile tests were conducted on the replicated samples of Ukam-Plant Fibre Reinforced CNSL Composite to obtain the optimum properties. Hounsfield Mosanto Tensometer, a universal testing machine was used to conduct tensile tests to determine the control factor levels quality characteristics considered necessary to optimize the mechanical property being investigated. Methodology of traditional and experimental design approach of Taguchi was used for the larger the better to obtain the highest signal to noise ratio (SN ratio) for the quality characteristic being investigated and also to correlate the control factors (fibre condition i.e. untreated, silane treated and alkaline treated, volume fraction and fibre size). Minitab 16 software was used for statistical analysis to fine-tune conclusions and produce quantitative estimates. The optimum settings were as follows: alkaline treatment, volume fraction of $50 \%$, and $50 \mathrm{~mm}$ of fibre size which resulted in 18.69MPa optimum tensile strength. The experimental results obtained agreed satisfactorily with the Minitab 16 software predictions. The mechanical property studied revealed that composite depends significantly on the reinforcement combination of control parameters.
\end{abstract}

Keywords: Cashew Nut Shell Liquid; Robust Design; Taguchi; Tensile Strength; Ukam-Plant Fibre

\section{Introduction}

Tensile strength or ultimate tensile strength is the maximum stress that a material can withstand while being pulled. It is also defined as a stress which is measured as force per unit area i.e. the highest point of the stress-strain curve. Natural fibres such as ukam-plant fibres present many advantages compared with synthetic fibres which make them attractive as reinforcements in composite materials. They come from abundant and renewable resources, which ensures a continuous supply and a significant material cost saving to the plastics industry. Unlike brittle fibres, such as glass and carbon fibres, natural fibres are flexible and will not fracture when processed over sharp curvatures [1]. Chemical modification of natural fibres such as ukam-plant fibres is usually applied to correct for deficiencies like hydrophilic nature of the fibres. Modification may result in improved performance of the composites produced. Apart from the hydrophilic nature of natural fibres, the properties of the natural fibre reinforced composites can also be influenced by fibre volume fraction, fibre size and orientation [2]. In general, high volume fraction is required to achieve high performance of the composite. Therefore, the effects of volume fraction on the mechanical properties of natural composite such as Ukam-Plant Fibres Reinforced CNSL Composite are particularly significant. It is often observed that

\footnotetext{
* Corresponding author: Godwin Kingsley Obiazi

Department of Mechanical Engineering, Nnamdi Azikiwe University, Awka. Nigeria. 
the increase in fibre volume fraction leads to an increase in tensile properties [3]. In general, the tensile properties of composites are distinctly improved by adding fibres to a polymer matrix, since fibres have much higher stiffness and strength values than those of the matrix. Higher volume fraction is desired for the purpose of achieving high performance of short fibre reinforced polymer composites. It is often observed that the presence of fibres or other reinforcement in the polymeric matrix raises the composite strength and modulus [4].

Taguchi's approach to parameter design is adopted in this paper in evaluating the ultimate tensile strength of UkamPlant Fibre Reinforced CNSL Composite material in other to know the optimum setting of fibre volume fraction and fibre size. Taguchi method was developed by Dr. Genichi Taguchi of Japan who maintained the variation in the 1980 s. He developed a method for designing experiments to investigate how different parameters affect the mean and variance of a process performance characteristic that defines how well the process is functioning. This approach provides researchers with a systematic and efficient method for determining near optimum design parameters for performance and cost [5]. The objective is to select the best combination of control parameters so that the composite is most robust with respect to noise factors. The Taguchi method utilizes orthogonal arrays for design of experiments theory to study a large number of variables with a small number of experiments. Using orthogonal array significantly reduces the number of experimental configurations to be studied. Furthermore, the conclusions drawn from small scale experiments are valid over the entire experimental region spanned by the control factors and their settings.

In this paper Ukam-Plant Fibre was used as a reinforcing material and cashew nut shell liquid used as matrix material to produce a composite material to evaluate the tensile strength at different reinforcement combination to obtain the optimum setting for recommendation for fabrication of auto-body.

\section{Literature/Background of the study}

Natural fibres have recently become attractive to researchers, engineers and scientists as viable alternatives to glass fibres either alone or combined in composite materials for various application. Due to their low cost, fairly good mechanical properties, high specific strength, non-abrasive, eco-friendly and biodegradability characteristics, they are exploited as a replacement for the conventional fibre, such as glass, aramid and carbon. Besides, natural fibres are expected to give less health problems for the people producing the composites. Natural fibres do not cause skin irritations and they are not suspected of causing lung cancer. The disadvantages are their relatively high moisture sensitivity and their relatively high variability of diameter and length. The abundance of natural fibres combined with the ease of their process ability is an attractive feature which makes it a covetable substitute for synthetic fibres that are potentially toxic. One of the factors that significantly influence the properties and interfacial characteristics of the composite are the processing parameters used. Therefore, suitable processing techniques and parameters must be carefully selected in order to yield the optimum composite products [6]. Okpanachi et al [7] studied the effects of fibre surface treatment on the mechanical properties of Ukam-Plant Fibre Reinforced Composites and they reported that cellulose fibres are generally incompatible with polymers due to their hydrophilic nature and that cellulose fibres can be enhanced, by the modification of polymeric matrix of the fibre surface using chemicals. The results revealed an improvement in the fibres adhesion characteristics of the ukam-plant fibres, the tensile strength of the fibre composite improved by $46 \%$ after silane and alkaline treatments. It was then concluded that fibres surface treatment have significant impact in the mechanical properties of natural fibres.

One of the special characteristics of natural fibre reinforced composites is that their properties can be stream lined to make different types of loading conditions and this study is aimed at exploiting this opportunity to determine the optimum tensile strength of Ukam-Plant Fibre Reinforced CNSL Composite employing Taguchi methodology for recommendation as optimum condition for the design of auto-body fabrication using the hand lay-up techniques.

\section{Materials and methods}

\subsection{Materials}

The materials needed for the composite fabrication are as follows: Untreated Ukam-Plant Fibres, Silane treated UkamPlant Fibres, Alkaline treated Ukam-Plant Fibres, catalyst which is an organic peroxide named methylethylketone peroxide, an accelerator named Cobalt derivative, this is used when cure at room temperature is required and casew nut shell Liquid as the reinforcement. 


\subsection{Methodology}

The methodology of this study relies on traditional design methods of Taguchi to optimize the ultimate tensile strength of Ukam-Plant Fibre Reinforced CNSL Composite. This method involves reducing the variation in a process through robust design of experiments. The overall objective of the method is to produce high quality product at low cost to the researchers.

\subsubsection{Ukam-Plant Fibres Extraction}

Ukam-plants were sourced from Nsukka in Enugu State. The preparation and laboratory work was carried out by JuNeng Nigeria Limited, Nsukka. The plant was soaked in water and left for about Twelve days before being washed to remove some non fibre contaminants. This is a process known as "aging". The extracted ukam-plant fibres were treated with two chemicals to investigate the variation in their properties after treatments and some left as untreated. The treatments are with silane solution and with sodium hydroxide (alkaline treatment). Silane solution contains $60 \%$ ethanol and $40 \%$ water mixed well and allowed to stand for an hour. The $\mathrm{pH}$ of the solution was carefully controlled to bring about complete hydrolysis. The $\mathrm{pH}$ of the solution was 9.0; after that, the solution was decanted and the fibres dried. The silane solution helps to dissolve out some impurities detracting to the resin; these impurities prevent good adhesion of the resin to the fibres. The extracted ukam plant fibres were also soaked in a solution containing $40 \mathrm{~g}$ of $\mathrm{NaOH}_{(\mathrm{s})}$ in 1 litre of water and left for about twenty four hours at room temperature; after that, the fibres were rinsed and dried under the sun so that the fibres were straight, this helps to dissolve out the lignin on the fibre, the lignin has a way of interacting with the resin in such a way as to cause de-lamination in the moulded work. After the treatment with sodium hydroxide, the entire fibres were alkaline in nature and so a dilute acid was applied for the neutralization. The fibres were washed finally with water containing a few drops of $\mathrm{H}_{2} \mathrm{SO}_{4}$ to remove the final traces of alkali; finally, the fibres were dried at room temperature.

\subsubsection{Cashew Nut Shell Liquid}

The cashew nut shell liquid (CNSL) is alkylphenolic oil contained in the spongy mesocarp of the cashew nut (anacardium occidentiallis). The main constituents of cashew nut shell liquid are cardonal, anacardic acid, cardol, 2-methylcardol and small amount of polymeric material. The CNSL used in this research work was extracted from cashew nuts at the chemistry laboratory of the University of Nigeria, Nsukka. Cashew Nut Shell Liquid was extracted using the "Solvent Extraction Method".

\subsubsection{Hand Lay-up Technique}

According to Richard Wood [8], hand lay-up or contact moulding technique is the simplest method used for the fabrication of composite and lends itself particularly to short run production of large mouldings such as car bodies. A single female mould is used to give a finished outer surface on the component. Before any catalyzed and accelerated resin is applied to the mould, its entire surface is waxed and coated, ideally with a release agent such as polyvinyl alcohol in order to ensure clean removal of the finished mould. The chopped fibres are impregnated with catalyzed and accelerated resins which are rolled by hand, using plastic roller to consolidate the reinforcement and to drive out any entrapped air. During the time of curing, a compressive pressure of $0.05 \mathrm{MPa}$ is normally applied on the mould. When the resin has gelled, but not fully cured, the surplus laminate is trimmed off to the edges of mould, the laminate allowed curing for $24 \mathrm{hrs}$ fully, after which it can be freed and lifted from the mould.

\subsubsection{Composite Fabrication}

The following procedures were adopted for the composite fabrication:

- Each of the untreated, silane treated and alkaline treated bundles of strands of ukam-plant fibres were cut into three lengths: $5 \mathrm{~mm}, 50 \mathrm{~mm}$ and $100 \mathrm{~mm}$. This process of fibres cutting preparation is known as 'chopping'.

- Considering each part from procedure (1) above, the fibres was collected for $10 \%, 30 \%$ and $50 \%$ volume fraction to determine the equivalent mass fraction of fibres and CNSL (resin) to use for the fabrication and applying Taguchi design of experiment matrix of table 2 below.

- Nine (9) mild steel moulds of dimension (300 x $300 \times 10) \mathrm{mm}$ were prepared, waxed and coated with a release agent (polyvinyl alcohol) in order to ensure clean removal of the mould after curing.

- Hand lay-up technique was employed in the production of the composite laminates used in this work as explained in sub-section 3.3 above in this paper. Considering each part from procedure (1) and applying procedure (2) above, the fibres was impregnated with catalyzed and accelerated CNSL (resin) which was 
rolled by hand using plastic roller and paint brush to consolidate the ukam-plant fibres and to drive out any entrapped air.

- A compressive pressure of 0.05MPa was applied on each of the mould.

- The Surplus laminates were trimmed off using sharp knife or scissor to the edges of the mould.

- $\quad$ The composite specimens were cured for 24 hours.

A total of nine (9) experiments were performed to form nine (9) laminate plates based on the design matrix of table 2 below. The tensile specimen of dimension ( $160 \times 19 \times 3.2) \mathrm{mm}$ was cut using the electric cutting machine according to ASTM D638M from the laminate plates formed above; replicate samples were also cut according to the design matrix of table 2.

Stress $=\sigma=\frac{F}{A_{0}}$

Strain $=\frac{L_{i}-L_{0}}{L_{0}}=\frac{\Delta L}{L_{0}}$

Where,

$\mathrm{F}=$ load or force in $(\mathrm{N})$

$\mathrm{A}_{0}=$ Area $=19 \times 3.2=60.8 \mathrm{~mm}^{2}$

$\mathrm{L}_{0}=$ Original length in $(\mathrm{mm})=160 \mathrm{~mm}$

$\Delta \mathrm{l}=$ change in length in $(\mathrm{mm})$

Table 1 Parameters and Levels for Mechanical Property Evaluation

\begin{tabular}{|l|c|c|c|}
\hline Control factors and Units & Low Level(1) & Medium Level (2) & High Level (3) \\
\hline A: Fibre condition & 1 (untreated) & 2 (silane treated) & 3(alkaline treated) \\
\hline B: Volume fraction (\%) & 10 & 30 & 50 \\
\hline C: fibre size (mm) & 5 & 50 & 100 \\
\hline
\end{tabular}

Table 2 Taguchi Standard Orthogonal Array L9

\begin{tabular}{|c|c|c|c|}
\hline $\begin{array}{l}\text { Experiment } \\
\text { Number }\end{array}$ & A: Fibre Condition & B: Volume Fraction (\%) & C: Fibre Size (mm) \\
\hline 1 & 1 & 1 & 1 \\
\hline 2 & 1 & 2 & 2 \\
\hline 3 & 1 & 3 & 3 \\
\hline 4 & 2 & 1 & 3 \\
\hline 5 & 2 & 2 & 1 \\
\hline 6 & 2 & 3 & 3 \\
\hline 7 & 3 & 1 & 1 \\
\hline 8 & 3 & 2 & 2 \\
\hline 9 & 3 & 3 & 2 \\
\hline
\end{tabular}




\subsubsection{Signal-to-Noise Ratio, Mean Response and Standard Deviation}

$S N_{L}=-10 \log _{10}\left(\frac{1}{N} \sum_{u=1}^{N_{i}} \frac{1}{y_{u}^{2}}\right)$

$\bar{y}_{i}=\left(\sum_{u=1}^{N_{i}} \frac{y_{i u}}{N_{i}}\right)$

$S_{i}=\sqrt{\sum_{u=1}^{N_{i}} \frac{\left(y_{i u}-\bar{y}_{i}\right)^{2}}{N_{i}-1}}$

Where

$i=$ experiment number

$u=$ trial number

$N_{i}=$ Number of trials for experiment i

$\bar{y}_{i}=$ mean value

$S_{i}=$ Standard deviation

$y_{i u}=$ value of the performance characteristic

\section{Results and discussion}

This section presents all the results and discussions that follow in this paper, here the results are displayed in tabular form and graphically, in addition, secondary variables are determined and displayed. The tensile strength of Ukam-Plant Fibre Reinforced CNSL Composite is being investigated for optimum reinforcement combinations to give the optimum setting by the application of Taguchi methodology. The signal to noise ratio and mean responses for the control factors are evaluated and presented, using Hounsfield Monsanto Tensometer.

Table 3 Summary Results for Tensile Test Experiment by the Application of Taguchi Robust Design using Microsoft excel

\begin{tabular}{|c|c|c|c|c|c|c|c|c|c|}
\hline \multirow{2}{*}{$\begin{array}{l}\text { Expt. } \\
\text { No }\end{array}$} & \multirow{2}{*}{$\begin{array}{l}\text { A: } \\
\text { Fibre } \\
\text { condition }\end{array}$} & \multirow{2}{*}{$\begin{array}{l}\text { B: } \\
\text { Volume } \\
\text { Fraction } \\
(\%)\end{array}$} & \multirow{2}{*}{$\begin{array}{l}\text { C: } \\
\text { Fibre } \\
\text { Size } \\
(\mathrm{mm})\end{array}$} & \multicolumn{3}{|c|}{ Tensile Response (MPa) } & \multirow{2}{*}{$\begin{array}{l}\text { Mean } \\
\text { Ultimate } \\
\text { Tensile } \\
\text { Response } \\
\text { (MPa) }\end{array}$} & \multirow[t]{2}{*}{ STD } & \multirow{2}{*}{$\begin{array}{l}\text { SN } \\
\text { Ratio }\end{array}$} \\
\hline & & & & Trial 1 & Trial 2 & Trial 3 & & & \\
\hline 1 & 1 & 10 & 5 & 13.1579 & 13.3224 & 13.1579 & 13.2127 & 0.09496 & 22.4194 \\
\hline 2 & 1 & 30 & 50 & 14.9671 & 14.8026 & 14.3092 & 14.6930 & 0.34238 & 23.3374 \\
\hline 3 & 1 & 50 & 100 & 15.2960 & 14.9671 & 15.1316 & 15.1316 & 0.164470 & 23.5967 \\
\hline 4 & 2 & 10 & 50 & 13.9803 & 14.1417 & 14.3092 & 14.1437 & 0.16448 & 23.0101 \\
\hline 5 & 2 & 30 & 100 & 14.1447 & 14.3092 & 14.4737 & 14.3092 & 0.16447 & 23.112 \\
\hline 6 & 2 & 50 & 5 & 15.9540 & 16.1184 & 16.2829 & 16.1184 & 0.16447 & 24.1455 \\
\hline 7 & 3 & 10 & 100 & 16.4474 & 16.9408 & 17.1053 & 16.8311 & 0.34238 & 24.5186 \\
\hline 8 & 3 & 30 & 5 & 17.2697 & 17.4342 & 17.5987 & 17.4342 & 0.16447 & 24.8273 \\
\hline 9 & 3 & 50 & 50 & 18.0921 & 18.4211 & 18.7500 & 18.4211 & 0.32895 & 25.3035 \\
\hline
\end{tabular}

Table 4 Presentation of SNratio, the Mean Response and Standard Deviation for Tensile Response using Microsoft excel

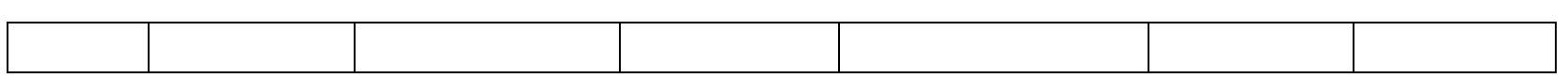




\begin{tabular}{|c|c|c|c|c|c|c|}
\hline $\begin{array}{l}\text { Expt. } \\
\text { No }\end{array}$ & $\begin{array}{l}\text { A:Fibre } \\
\text { condition }\end{array}$ & $\begin{array}{l}\text { B: Volume } \\
\text { Fraction (\%) }\end{array}$ & $\begin{array}{l}\text { C: } \\
\text { Size (mm) }\end{array}$ & $\begin{array}{l}\text { Fibre } \\
\text { Mean Tensile } \\
\text { Response (MPa) }\end{array}$ & $\begin{array}{l}\text { Standard } \\
\text { Deviation }\end{array}$ & SNi ratio \\
\hline 1 & 1 & 10 & 5 & 13.2127 & 0.09496 & 22.4194 \\
\hline 2 & 1 & 30 & 50 & 14.6930 & 0.34238 & 23.3374 \\
\hline 3 & 1 & 50 & 100 & 15.1316 & 0.16447 & 23.5967 \\
\hline 4 & 2 & 10 & 50 & 14.1437 & 0.16448 & 23.0101 \\
\hline 5 & 2 & 30 & 100 & 14.3092 & 0.16447 & 23.1112 \\
\hline 6 & 2 & 50 & 5 & 16.1184 & 0.16447 & 24.1455 \\
\hline 7 & 3 & 10 & 100 & 16.8311 & 0.34238 & 24.5186 \\
\hline 8 & 3 & 30 & 5 & 17.4342 & 0.16447 & 24.8273 \\
\hline 9 & 3 & 50 & 50 & 18.4211 & 0.32895 & 25.3035 \\
\hline
\end{tabular}

Table 5 Presentation of Averages of Signal-to-Noise Ratio Responses for factor A (Fibre condition) at levels 1, 2, 3 within experiments 1 to 9

\begin{tabular}{|c|l|l|}
\hline Factor level & Average of responses for different experiments & Response Value \\
\hline 1 & $\frac{\left(S N_{1}+S N_{2}+S N_{3}\right)}{3}$ & 23.12 \\
\hline 2 & $\frac{\left(S N_{4}+S N_{5}+S N_{6}\right)}{3}$ & 23.42 \\
\hline 3 & $\frac{\left(S N_{7}+S N_{8}+S N_{9}\right)}{3}$ & $\underline{24.88}$ \\
\hline
\end{tabular}

\subsection{Averages of the Signal to Noise Ratio for Tensile Response}

Table 6 The Response for the Signal to Noise Ratio Averages

\begin{tabular}{|c|c|c|c|}
\hline Level & A: Fibre Condition & B: Volume Fraction (\%) & C: Fibre Size (mm) \\
\hline 1 & 23.12 & 23.32 & 23.80 \\
\hline 2 & 23.42 & 23.76 & $\underline{23.88}$ \\
\hline 3 & $\underline{24.88}$ & $\underline{24.35}$ & 23.74 \\
\hline Delta & 1.77 & 1.03 & 0.14 \\
\hline Rank & 1 & 2 & 3 \\
\hline
\end{tabular}




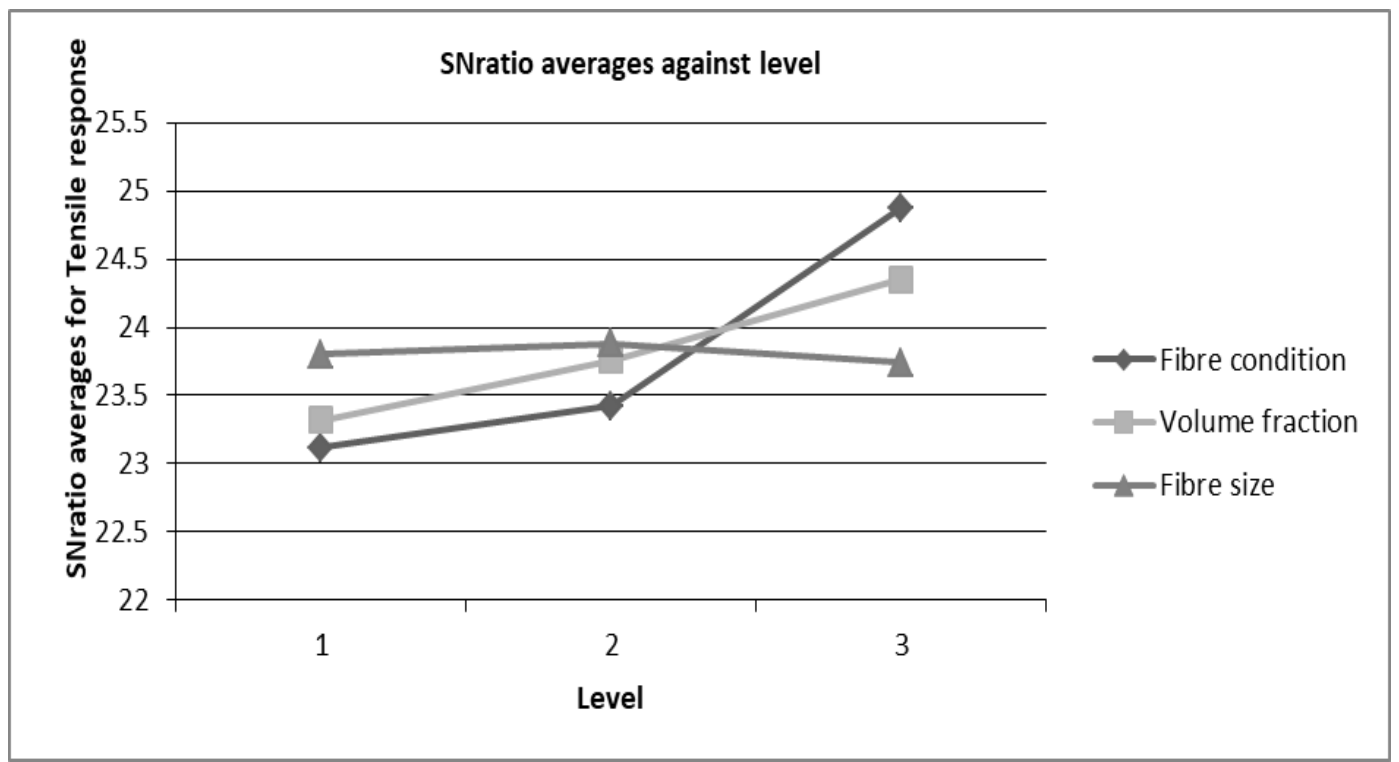

Figure 1 Plot of SNratios Averages for Tensile Response using Microsoft excel

\subsection{Averages of the Mean Response for Tensile Strength}

Table 7 Presentation of Averages of mean Responses for factor A (Fibre condition) at levels 1, 2, 3 within experiments 1 to 9

\begin{tabular}{|c|l|l|}
\hline Factor level & $\begin{array}{l}\text { Average of responses for different } \\
\text { experiments }\end{array}$ & Response Value \\
\hline 1 & $\frac{\left(\bar{y}_{1}+\bar{y}_{2}+\bar{y}_{3}\right)}{3}$ & 14.35 \\
\hline 2 & $\frac{\left(\bar{y}_{4}+\bar{y}_{5}+\bar{y}_{6}\right)}{3}$ & 14.86 \\
\hline 3 & $\frac{\left(\bar{y}_{7}+\bar{y}_{8}+\bar{y}_{9}\right)}{3}$ & $\underline{17.56}$ \\
\hline
\end{tabular}

Table 8 Summary of Mean of Means of Tensile Strength Response using Microsoft excel

\begin{tabular}{|c|c|c|c|}
\hline Level & A: Fibre Conditions & B: Volume Fraction (\%) & $\begin{array}{c}\text { C: Fibre Size } \\
\text { (mm) }\end{array}$ \\
\hline 1 & 14.35 & 14.73 & 15.59 \\
\hline 2 & 14.86 & 15.48 & $\underline{15.75}$ \\
\hline 3 & $\underline{17.56}$ & $\underline{16.56}$ & 15.42 \\
\hline Delta & 3.22 & 1.83 & 0.33 \\
\hline Rank & 1 & 2 & 3 \\
\hline Average & 15.59 & 15.59 & 15.59 \\
\hline
\end{tabular}




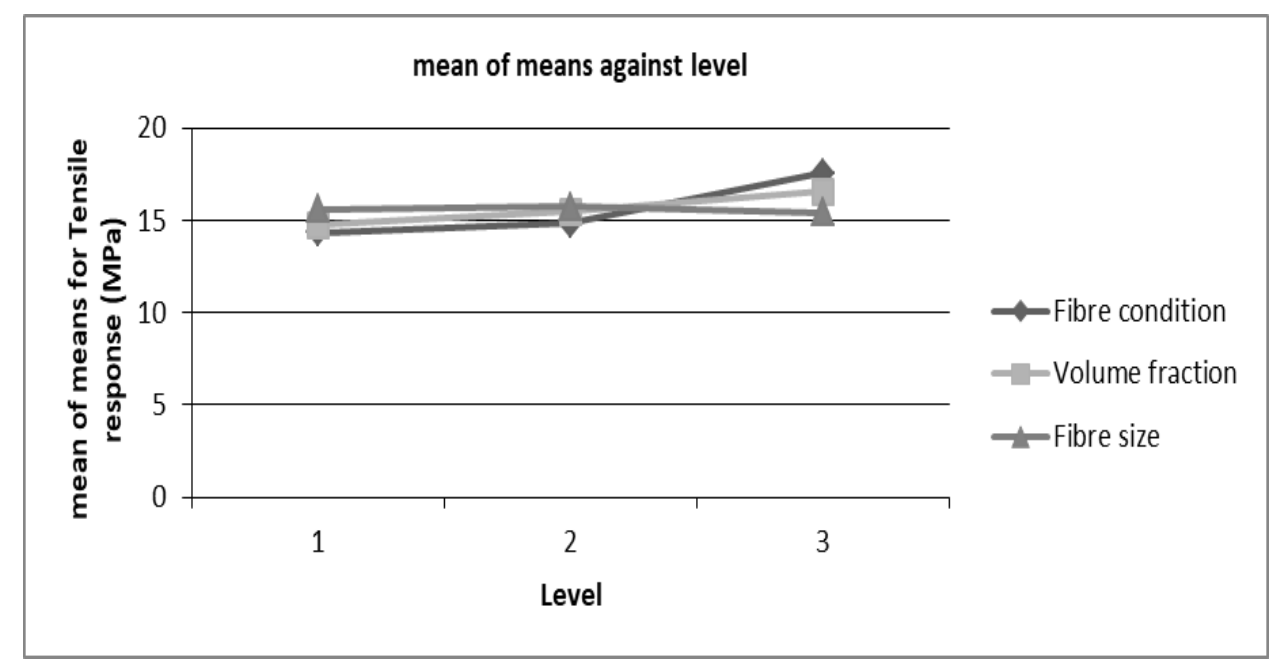

Figure 2 Plots of Mean of Means against Level for Tensile Response using Microsoft excel

Table 9 Optimum Factor Setting and Expected Optimum Tensile Strength of Composite

\begin{tabular}{|c|c|c|c|}
\hline Control Factor & Optimum Level & Optimum Setting & Expected Response \\
\hline A & 3 (High) & (3) Alkaline $(\mathrm{NaOH})$ & \\
\hline B & 3 (High) & $50 \%$ & $18.69 \mathrm{MPa}$ \\
\hline C & 2 (Medium) & $50 \mathrm{~mm}$ & \\
\hline
\end{tabular}

Table 10 Response Table for Signal to Noise Ratio Using Minitab 16 Software for Tensile Strength

\begin{tabular}{|c|c|c|c|}
\hline Level & A: Fibre Conditions & $\begin{array}{l}\text { B: Volume Fraction } \\
\text { (\%) }\end{array}$ & C: Fibre Size (mm) \\
\hline 1 & 23.11 & 23.31 & 23.79 \\
\hline 2 & 23.41 & 23.75 & $\underline{23.87}$ \\
\hline 3 & $\underline{24.87}$ & $\underline{24.34}$ & 23.73 \\
\hline Delta & 1.76 & 1.03 & 0.14 \\
\hline Rank & 1 & 2 & 3 \\
\hline
\end{tabular}

Table 11 Response Table for Mean of Means Using Minitab 16

\begin{tabular}{|c|c|c|c|}
\hline Level & A: Fibre Conditions & B: Volume Fraction (\%) & C: Fibre Size (mm) \\
\hline 1 & 14.34 & 14.72 & 15.59 \\
\hline 2 & 14.85 & 15.47 & $\underline{15.74}$ \\
\hline 3 & $\underline{17.55}$ & $\underline{16.55}$ & 15.42 \\
\hline Delta & 3.21 & 1.83 & 0.30 \\
\hline
\end{tabular}




\begin{tabular}{|l|l|l|l|}
\hline Rank & 1 & 2 & 3 \\
\hline
\end{tabular}
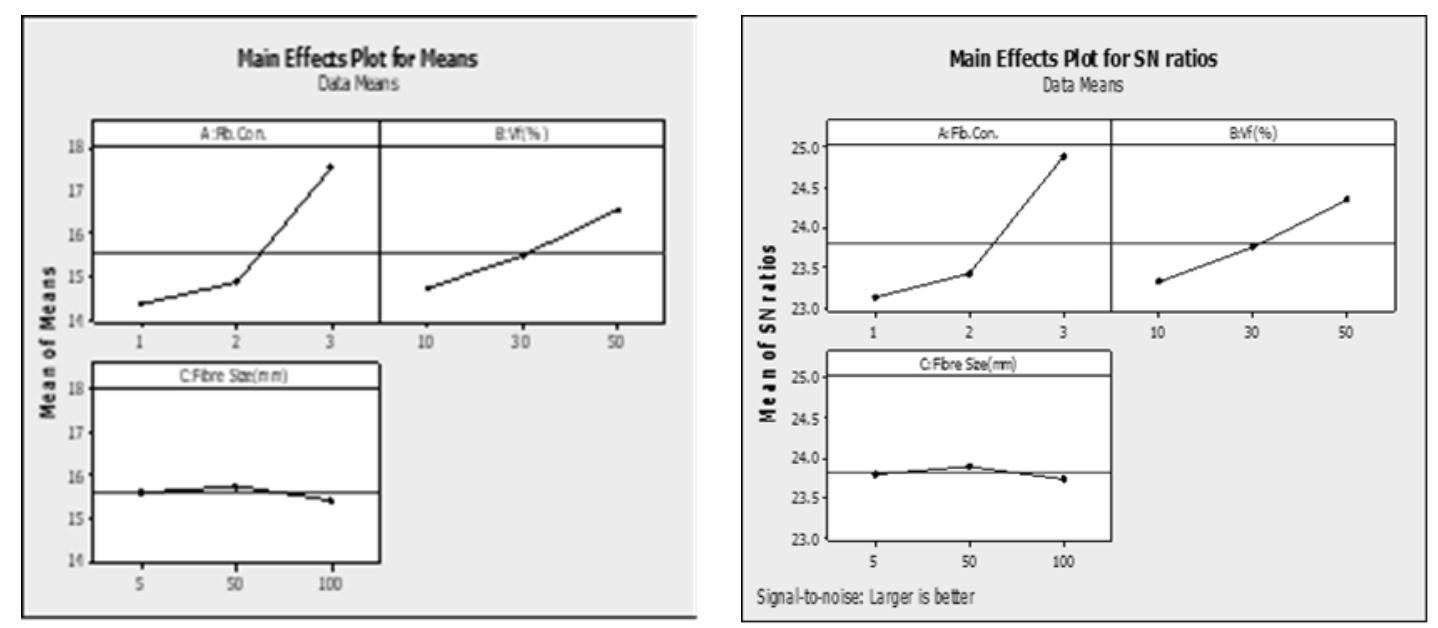

Figure 3 Main Effects Plots for SN ratios and Means for Tensile Response using Minitab 16

\subsection{Regression Analysis for Tensile Strength Response}

ultimate tensile strength $=11.1+1.61 A+0.0457 B-0.00182 C$

Where, $\mathrm{A}, \mathrm{B}$ and $\mathrm{C}$ are individual levels of factors

Table 12 Optimum Factor Setting and Predicted Expected Optimum Tensile Strength of Composite using Minitab 16

\begin{tabular}{|c|c|c|c|}
\hline Control Factor & $\begin{array}{c}\text { Optimum } \\
\text { Level }\end{array}$ & Optimum Setting & $\begin{array}{c}\text { Predicted } \\
\text { Response }\end{array}$ \\
\hline $\mathrm{A}$ & 3 (High) & (3) Alkaline $(\mathrm{NaOH})$ & \\
\hline $\mathrm{B}$ & 3 (High) & $50 \%$ & $18.124 \mathrm{MPa}$ \\
\hline $\mathrm{C}$ & 2 (Medium) & $50 \mathrm{~mm}$ & \\
\hline
\end{tabular}

Table 13 Regression Analysis for Tensile Strength Response

\begin{tabular}{|l|c|c|c|c|}
\hline \multicolumn{1}{|c|}{ Predictor } & Coef & SE Coef & T & P \\
\hline Constant & 11.0952 & 0.9089 & 12.21 & 0.000 \\
\hline A:Fibre Condition & 1.6082 & 0.3194 & 5.04 & 0.004 \\
\hline B: Volume Fraction (\%) & 0.04570 & 0.01597 & 2.86 & 0.035 \\
\hline C:Fibre Size (mm) & -0.001821 & 0.006721 & -0.27 & 0.797 \\
\hline S $=0.782313 \quad$ R-Sq=87.1\% R - Sq(adj) $=79.3 \%$ \\
\hline
\end{tabular}

Table 14 Analysis of Variance for Tensile Strength Response

\begin{tabular}{|l|l|l|l|l|l|}
\hline Source & DF & SS & MS & F & P \\
\hline
\end{tabular}




\begin{tabular}{|l|c|c|c|c|c|}
\hline Regression & 3 & 20.5739 & 6.8580 & 11.21 & 0.012 \\
\hline Residual Error & 5 & 3.0601 & 0.6120 & & \\
\hline Total & 8 & 23.6340 & & & \\
\hline
\end{tabular}
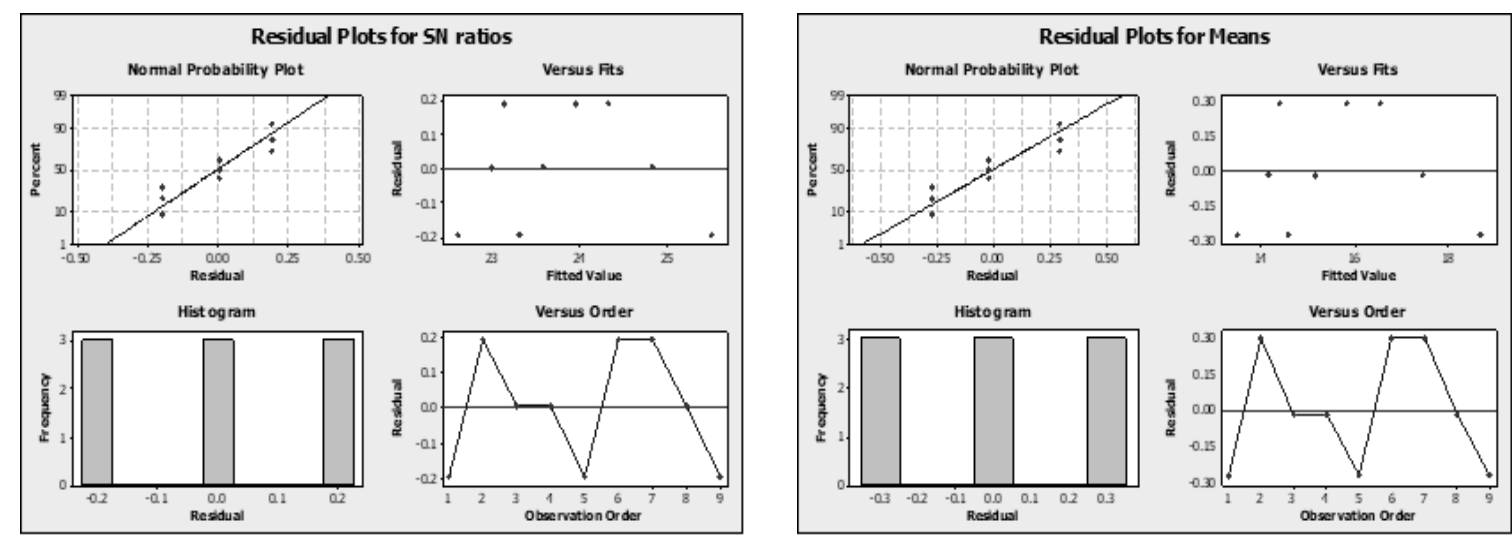

Figure 4 Residual Plots for SNratios and Means for Tensile Response

\subsection{Discussion on the ultimate tensile strength based on Taguchi Design of Experiments}

After the trials experiments had been performed using Hounsfield Mosanto Tensometer applying Taguchi design of experiments, we then determined the effect each variable had on the output by evaluating the signal-to-noise ratio as shown in table 3 above. A greater $\mathrm{SN}_{\text {ratio }}$ average value corresponds to a better performance. Therefore, based on the signal to noise ratio analysis of table 6 and fig 1 above, it was observed that optimum setting for parameter A: fibre condition is level 3 (High), the optimum setting for parameter B: volume fraction is level 3 (High) and the optimum setting for parameter C: fibre size is level 2 (Medium) as shown in table 9 above, i.e. increasing the volume fraction increases the ultimate tensile strength of the material which is desirable and recommended for auto-body construction. With table 8 and fig 2 above, it was observed that the averages for the mean of means for the ultimate tensile response for each factor for each level, and the plot of mean of means against level respectively, in addition to optimum setting which was already determined and summarized in table 9, the expected optimum response was computed employing Radharamanan \& Ansui model [9] as shown in table 9 above as $18.69 \mathrm{MPa}$. This is the optimum tensile strength of the material.

Minitab 16 was used for the statistical analysis. The results of the Minitab output are given in Tables 10 to 14 above. The $P$ values were used as a tool to check the significance of each of the coefficients, which in turn were necessary to understand the pattern of the mutual interactions between the control parameters. The regression equations, $\mathrm{P}$ values, $\mathrm{F}$ values, $\mathrm{T}$ values, coefficient of determination, R-sq and adjusted coefficient of determination, R-sq (adj.) are given in subsection 4.3 and tables $4.20 \& 4.21$ above. The regression equation of subsection 4.3 above can be used to estimate the value of ultimate tensile strength of the composite with different factor levels, it is a predictor equation. The R-sq value stipulates that the predictors explain $87.1 \%$ of the variance in tensile strength. The adjusted $\mathrm{R}-\mathrm{sq}$ is $79.3 \%$ which accounts for the number of predictors in the model, the larger the magnitude of F-value and the smaller the P-values, the higher the significance of corresponding coefficient. The P-value in the analysis of variance table 14 (0.012) shows that the model estimated by the regression procedure is significant at significance level of 0.05 . The fitness of the model equations was expressed by the coefficient of determination; R-sq. R-sq indicates the fraction of the total variables of response variable that has been explained by the predicator variables, the greater the value of R-sq, the better the fit and the more effective the estimated regression equation for estimations and prediction finally, the greater the magnitude of a T-value, the greater the relative accuracy of estimating the corresponding coefficients. The results of the ANOVA presented in tables $13 \& 14$ indicate that the linear models are adequate and the coefficients are significant except for fibre size. The values obtained using Microsoft excels based on Taguchi methodology agreed totally with values predicted using Minitab 16 i.e. they show a satisfactory fit.

Fig 4 above is known as a residual plot which shows a graph of residuals against the independent variables on the horizontal axis. If the points in a residual plot are randomly dispersed, around the horizontal axis, a linear regression model is appropriate for the data, otherwise, a non-linear model is more appropriate. The residual plot of fig 6 actually shows that the linear model of section 4.3 in this paper is well suited as the residuals are randomly distributed. 


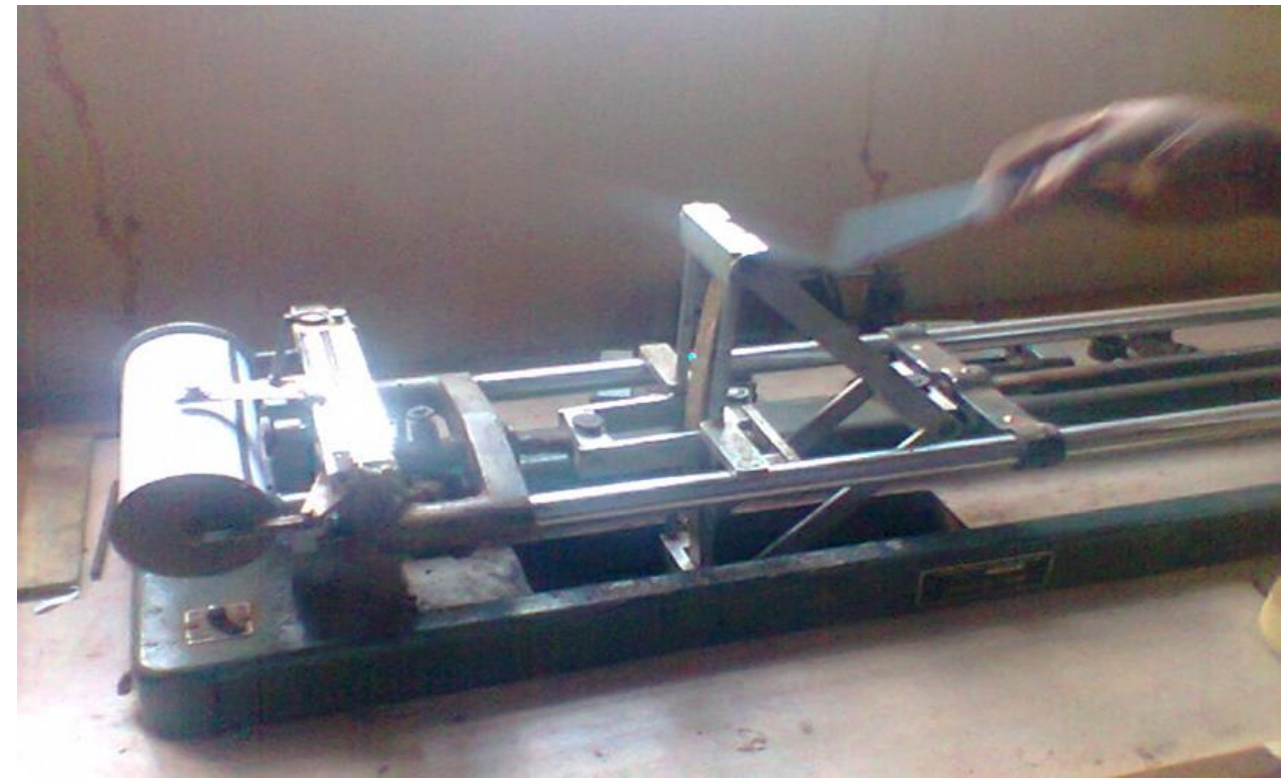

Figure 5 Mosanto Tensometer Model No: S/N 8889

\section{Conclusion}

The ultimate tensile strength of Ukam-Plant Fibre Reinforced CNSL Composite for fabrication of auto-body has been comprehensively studied with the following conclusions:

- An overview of Taguchi method has been presented and steps involved in the method were briefly described; it has shown that it is a sufficient and efficient approach for determining the optimum configuration of design parameters for performance quality and cost.

- Ukam-Plant Fibre Reinforced CNSL Composite has the optimum tensile strength of 18.69MPa when the control factors (fibre condition, volume fraction of fibres and fibre size) are set at 3(alkaline treatment), 50\% and $50 \mathrm{~mm}$ respectively.

- The ultimate tensile strength of Ukam-Plant Fibre Reinforced CNSL Composite depends significantly on the reinforcement combinations of control factors.

- Alkaline treated fibres are better than silane treated fibres for better ultimate tensile strength of Ukam-Plant Fibre Reinforced CNSL Composite and silane treated better than untreated fibres.

- The study can be extended using other methodology and results compared with these findings.

\section{Compliance with ethical standards}

\section{Acknowledgments}

The authors would like to acknowledge the services of Department of Civil Engineering, University of Nigeria, Nsukka for the use of their laboratory for the destructive test of this study, JuNeng Nigeria Limited Laboratory, Management of Standard Organization of Nigeria, Enugu state. We are also grateful to all staff (Academic and Non Academic) of Mechanical Engineering department, Nnamdi Azikiwe University, Awka, for their contributions.

\section{Disclosure of conflict of interest}

There are no conflict of interest for this research.

\section{References}

[1] Crawford, R.J., Plastic Engineering, 3rd edition, Elsevier, 2002.

[2] http://www.trafficcalming.net/speed-humps.htm 
[3] Ahmad I, Baharum A, Abdullah I. Effect of Extension Rate and Fibre loading on Mechanical Properties of Twaron Fibre Thermoplastic Natural Rubber Composite, Journal of Reinforced Plastic and Composites. 2006; 25 : 965.

[4] Li X, Tabil LG, Panigrahi S, Crerar WJ. The Influence of Fibre Content on Properties of Injection molded Flax Fibre HDPE Biocomposite, Canadian Biosystems Engineering. 2009; 1-10.

[5] Taguchi G. Taguchi on Robust Technology Development Method, ASME Press, New York. 1993; 1 - 40.

[6] Saheb DN, Jog P. Natural Fibre Polymer composites: A review, Advanced Polymer Technology. 1999; 18: $351-68$.

[7] Okpanachhi GE, Ogakwu P. Effects of Fibre surface treatment on the Machanical properties of Ukam Fibre Reinforced Polyester Composite, Journal of Engineering and Applied sciences. December 2012; 2: 1. 92.

[8] Richard W. Car Bodywork in Glass Reinforced Plastics, Pentech Press. 1980.

[9] Radharamanan R, Ansui AP. Quality Improvement of a Production Process using Taguchi Method, Proceedings of Institute of Industrial Engineers Annual Conference, Dollas, Texas. 2001; 20-22. 